\title{
CORONARY INVOLVEMENT AS KEY VASCULAR MANIFESTATION IN BEHÇET'S SYNDROME: A REPORT OF TWO CASES
}

Aline Carolina Capellato Dias Baccaro, ${ }^{1, \star}$, Leandro Lara do Prado ${ }^{1}$, Lucas Brandão Araujo da Silva ${ }^{1}$, Matheus Santos Rodrigues Silva ${ }^{1}$, Carla Baleeiro Rodrigues Silva ${ }^{1}$, Rodrigo da Silveira Vasconcelos ${ }^{1}$

1.Universidade de São Paulo, São Paulo (SP), Brazil.

*Corresponding author: aline.capellato@gmail.com

\section{BACKGROUND}

Behçet's syndrome (BS) is a heterogeneous systemic inflammatory disease, where, in addition to the typical picture of recurrent oral and genital ulcers, vascular involvement can affect vessels of any size or type (arteries, veins and capillaries), resulting in aneurysms and thrombosis. Cardiac involvement in BS is uncommon and diverse, with pericarditis being the most frequent presentation. Coronary involvement is rare and potentially fatal, and may present as myocardial infarction, arrhythmias, coronary aneurysm or coronary arteritis. Here we present two cases in which coronary involvement was the main feature that triggered BS diagnosis.

\section{CASE REPORT}

A 48-year-old male with previously controlled hypertension, presenting recurrent oral and genital ulcers, since he was 20 years old, right iliac artery aneurysm (with multiple surgical corrections), three episodes of deep venous thrombosis (one of them with anticoagulation), recurrent thrombophlebitis and pulmonary embolism (PE). Admitted with acute myocardial infarction with ST segment elevation, he was treated with stent angioplasty. Afterwards, he developed a stent thrombosis despite warfarin and dual antiplatelet therapy. Considering this atypical manifestation, rheumatology team evaluation was requested and BS diagnosis was instituted. Immunosuppression with monthly pulse cyclophosphamide and oral glucocorticoids was indicated. A 14 years-old male, with a history of recurrent oral ulcers in the last 7 years presented with ventilatory chest pain, thrombophlebitis of bilateral cephalic veins and a heterogeneous image adhered to the right ventricle measuring $5.1 \times 4.2 \mathrm{~cm}$. Chest computed tomography angiography confirmed PE and coronary computed tomography angiography showed a thrombosed aneurysm at the anterior descending coronary artery. After rheumatology team evaluation, BS diagnosis was made and immunosuppression with monthly pulse cyclophosphamide was instituted, associated with oral glucocorticoids. Both patients improved after immunosuppression, currently in outpatient follow-up, with controlled disease activity on azathioprine as a maintenance immunosuppressant, after 12 months of cyclophosphamide therapy.

\section{CONCLUSION}

Coronary involvement in BS is an uncommon and challenging manifestation. Thorough retrospective investigation of BS symptoms is essential. In this scenario, anticoagulation is controversial, but immunosuppression is mandatory, with a satisfactory response. 